\title{
Vlasov simulations of collisionless magnetic reconnection without background density
}

\author{
H. Schmitz and R. Grauer \\ Theoretische Physik I, Ruhr-Universität Bochum, 44780 Bochum, Germany
}

\begin{abstract}
A standard starting point for the simulation of collisionless reconnection is the Harris equilibrium which is made up of a current sheet that separates two regions of opposing magnetic field. Magnetohydrodynamic simulations of collisionless reconnection usually include a homogeneous background density for reasons of numerical stability. While, in some cases, this is a realistic assumption, the background density may introduce new effects both due to the more involved structure of the distribution function or due to the fact that the Alfvèn speed remains finite far away from the current sheet. We present a fully kinetic Vlasov simulation of the perturbed Harris equilibrium using a Vlasov code. Parameters are chosen to match the Geospace Environment Modeling (GEM) Magnetic Reconnection Challenge but excluding the background density. This allows to compare with earlier simulations [Schmitz, Grauer, Phys. Plasmas 13 (2006) 092309] which include the background density. It is found that the absence of a background density causes the reconnection rate to be higher. On the other hand, the time until the onset of reconnection is hardly affected. Again the off diagonal elements of the pressure tensor are found to be important on the $\mathrm{X}$-line but with modified importance for the individual terms.
\end{abstract}

Key words:

02.70.-c 52.25.Dg 52.65.Ff 52.25.Xz

\section{Introduction}

Magnetic reconnection is widely believed to be the most important process for converting magnetic energy into kinetic energy of the plasma. It is relevant for both space and laboratory plasmas and thought to be the main player for energy release in solar flares, coronal mass ejections and substorms in the earth's magnetosphere.

The models used to investigate collisionless magnetic reconnection usually start from the Harris-equilibrium. In this equilibrium two regions of oppositely oriented magnetic field lines are separated by a current sheet. A small perturbation of this equilibrium leads 
to a localised narrowing of the current sheet until the field lines reconnect on the $\mathrm{X}$-line. In the ideal MHD model, the frozen field condition prohibits such a reconnection. Therefore the reconnection process depends on a nonideal mechanism that breaks the frozen field condition. In the last years it has become apparent that the Hall-MHD framework provides a minimum model in understanding the fast reconnection (see eg. $[1,2,3]$ ). The Hall-Term itself, however, cannot provide this mechanism, since the field lines are still frozen in the electron flow. Due to symmetry constraints, only the electron inertia and the off-diagonal terms of the pressure tensor can provide a nonideal mechanism on the $\mathrm{X}$-line itself (see e.g Refs. [4,5,6,7]).

In this study, we use a Vlasov code to investigate collisionless reconnection without background density which is relevant, for example, for magnetotail reconnection. Apart from the background density, all other parameters are identical to the GEM setup to allow comparison. In the next section we briefly present the methods used in our investigation. The setup including the initial conditions and the boundary conditions is described in section 3. In section 4 we discuss the results. Separate subsections are dedicated to the discussion of the contributions of the terms in Ohm's law, especially the off-diagonal components of the pressure tensor, and to the detailed discussion of the electron distribution function. Section 5 will give a summary and present conclusions.

\section{Methods}

The kinetic description starts from the distribution functions $f_{k}(\mathbf{x}, \mathbf{v}, t)$ of species $k$, where $k=i, e$ denotes ions or electrons. The time development of the distribution function is described by the Vlasov equation

$$
\frac{\partial f_{k}}{\partial t}+\mathbf{v} \cdot \nabla f_{k}+\frac{q_{k}}{m_{k}}(\mathbf{E}+\mathbf{v} \times \mathbf{B}) \cdot \nabla_{\mathbf{v}} f_{k}=0 \text {. }
$$

Here $q_{k}$ and $m_{k}$ are the charge and the mass of the particles of species $k$. The Vlasov equation describes the incompressible flow of the species phase space densities under the influence of the electromagnetic fields.

The electromagnetic fields are solved using the Darwin approximation (see, for example Refs. $[8,9])$. The elimination of the vacuum modes in the Darwin approximation allows larger time-steps in the simulation since only the slower non-relativistic waves have to be resolved.

To close the set of equations of the Vlasov-Darwin system the charge density $\rho$ and the current density $\mathbf{j}$ have to be calculated from the distribution function,

$$
\rho=\sum_{k} q_{k} \int f_{k}(\mathbf{x}, \mathbf{v}) d^{3} v, \quad \quad \mathbf{j}=\sum_{k} q_{k} \int \mathbf{v} f_{k}(\mathbf{x}, \mathbf{v}) d^{3} v
$$

We use a $2 \frac{1}{2}$-dimensional Vlasov-code described in Refs. $[9,10]$. The term $2 \frac{1}{2}-$ dimensional means, we restrict the simulations to 2 dimensions in space but include all three velocity dimensions. The integration scheme is based on a flux conservative and positive scheme [11] which obeys the maximum principle and suffers from relatively little numerical diffusion. 


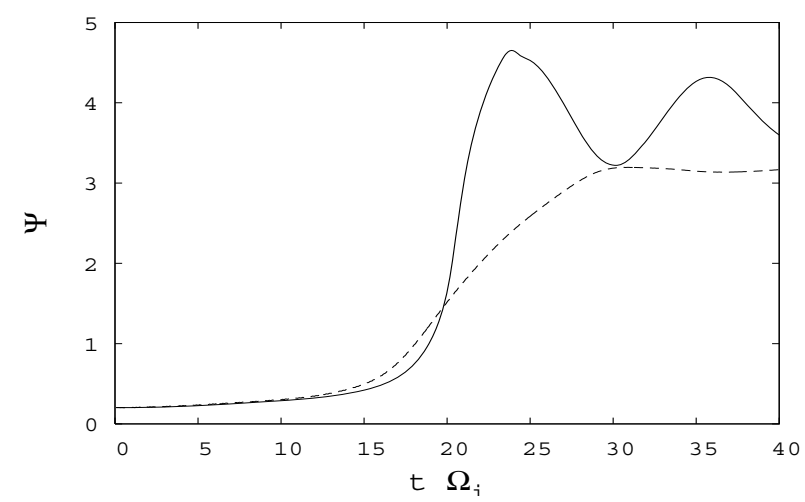

Fig. 1. Time evolution of the reconnected magnetic flux $\Psi$ throughout the simulation run without background density (solid line) and with background density (dashed line).

\section{Setup}

The reconnection setup is identical to the parameters of the GEM magnetic reconnection challenge [1]. The initial conditions are based on the Harris sheet equilibrium [12] in the $x, y$-plane

$$
\mathbf{B}(y)=B_{0} \tanh \left(\frac{y}{\lambda}\right) \hat{\mathbf{x}} .
$$

The particles have a shifted Maxwellian distribution with constant electron and ion temperatures $T_{i, e}$ and constant electron and ion drift velocities $V_{0 i, e}$. The density distribution is then given by $n_{0}(y)=n_{0} \operatorname{sech}^{2}(y / \lambda)$.

The total system size is $L_{x}=25.6 \lambda_{i}$ by $L_{y}=12.8 \lambda_{i}$, where $\lambda_{i}$ is the ion inertial length. Because of the symmetry constraints we simulate only one quarter of the total system size: $0 \leq x \leq L_{x} / 2$ and $0 \leq y \leq L_{y} / 2$. The sheet half thickness is chosen to be $\lambda=0.5 \lambda_{i}$. The temperature ratio is $T_{e} / T_{i}=0.2$ and a reduced mass ratio of $m_{i} / m_{e}=25$ is used. The simulation is performed on $256 \times 128$ grid points in space for the quarter simulation box. This corresponds to a resolution of $512 \times 256$. This implies a grid spacing of $\Delta x=\Delta y=0.05 \lambda_{i}$. The resolution in the velocity space was chosen to be $30 \times 30 \times 30$ grid points. The simulation was performed on a 32 processor Opteron cluster and took approximately 150 hours to complete.

An initial perturbation

$$
\psi(x, y)=\psi_{0} \cos \left(2 \pi x / L_{x}\right) \cos \left(\pi y / L_{y}\right)
$$

is added to the magnetic vector potential component $A_{z}$. To place the system directly into the nonlinear regime, the magnitude of the perturbation is chosen to be $\psi_{0}=0.1 B_{0} / \lambda_{i}$. We are not interested in the linear growth of the instability, but rather in the nonlinear reconnection phase that follows.

\section{Simulation Results}

Figure 1 shows the reconnected flux $\Psi=\int_{O}^{X} B_{y} d x$ against time throughout the simulation. For comparison, the flux for the simulation with a background density $n_{\infty}=0.2$ 

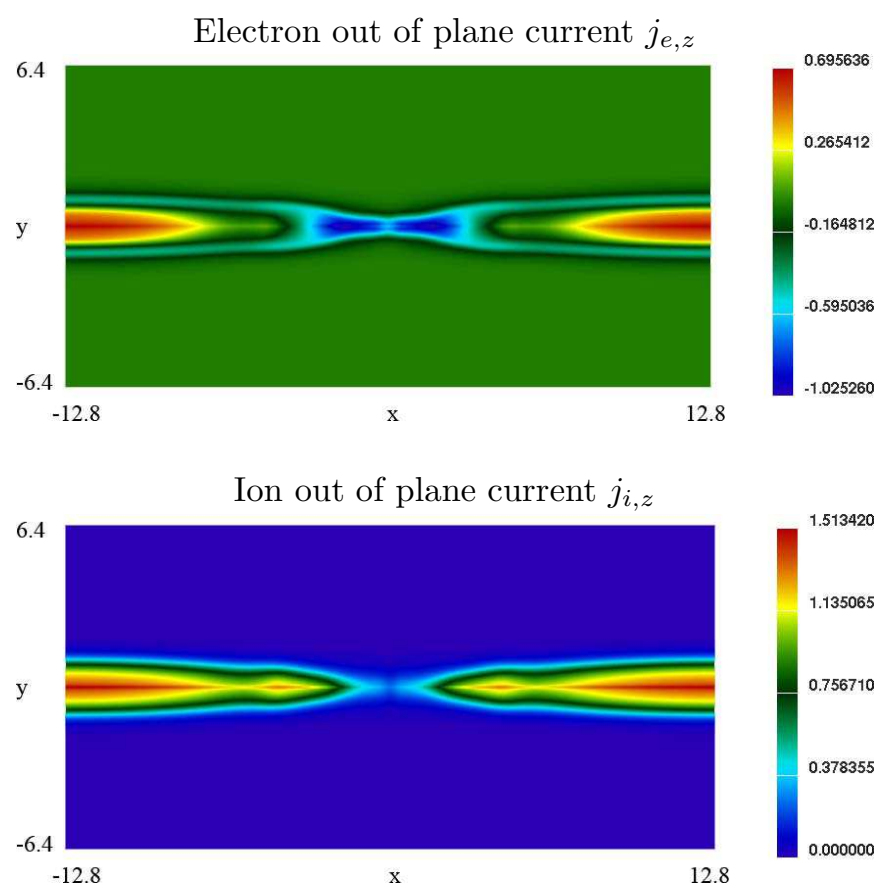

Fig. 2. The electron out of plane current $j_{e, z}$ (upper panel) and the ion out of plane current $j_{i, z}$ (lower panel) at time $\Omega_{i} t=18.9$

(see Ref [10]) is also shown. One can observe that the onset of the reconnection takes place roughly at the same time for both cases, although it is slightly delayed for the $n_{\infty}=0$ case. In the further development the $n_{\infty}=0$ case shows a reconnection which is much faster than with a background density. This can be understood by the increase of the Alfvèn speed as the density is reduced. In addition, one can observe oscillations of the reconnected flux for the $n_{\infty}=0$ case after the fast reconnection phase. The average value during the first oscillations is higher than the highest value of the $n_{\infty} \neq 0$ case. When reconnection stops, the plasma is almost completely trapped in the magnetic island. During reconnection the ions are accelerated towards the centre of the island $(\mathrm{O}-$ point). After reconnection stops the ions overshoot due to their inertia and the island starts oscillating. This oscillation is visible in the reconnected flux for times $t \Omega_{i} \geq 25$.

Figure 2 shows the out of plane electron and ion current densities. The figures are plotted at time $\Omega_{i} t=18.9$, when the reconnected flux reaches a value of $\Psi=1$. The features here are similar to the case with background density [10] but some differences can be seen. The electron current density is decreased on the X-line compared to the adjacent regions in the diffusion region. This dip in the electron current is largely due to the lack of current carrying electrons. Both electron and ion density are almost zero on the $\mathrm{X}$-line. Also the electron current along the separatrix is not as pronounced as in the $n_{\infty}=0.2$ case. Here the lack of current carrying electrons at the edges of the magnetic island is responsible. Finally the lower electron density outside the island is also the reason for a lower value of the quadrupolar out of plane magnetic field $B_{z}$ (not shown). The maximum value of $B_{z}$ at time $\Omega_{i} t=18.9$ in this simulation is about 0.064 


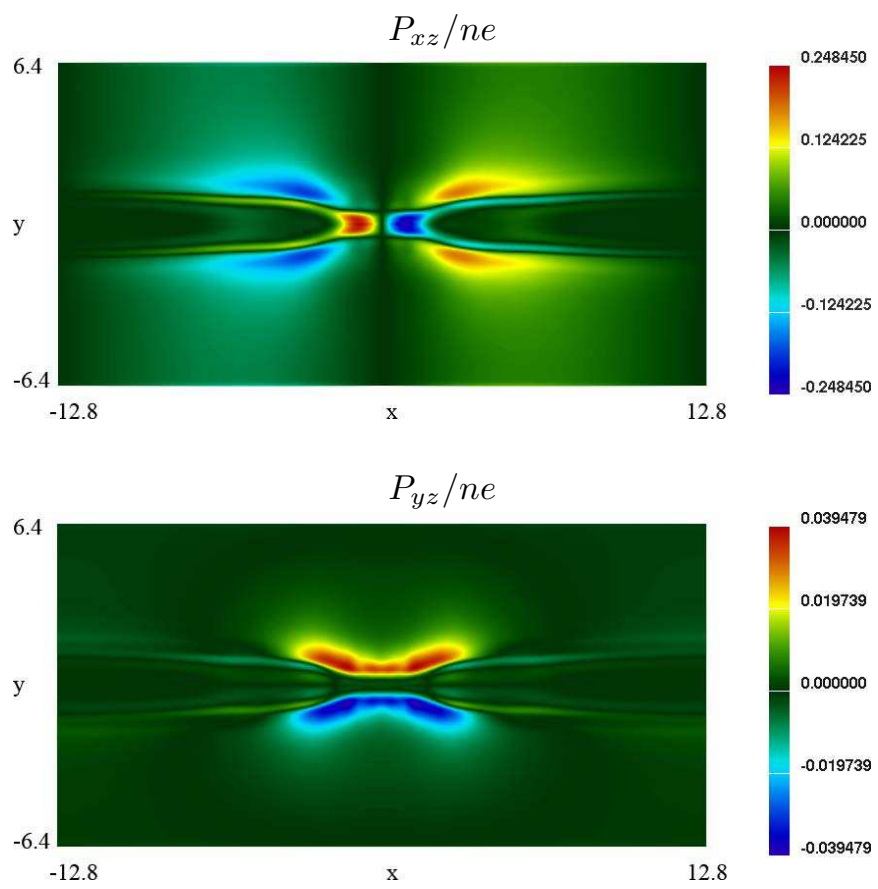

Fig. 3. The off-diagonal components $P_{x z}$ and $P_{y z}$ of the pressure tensor divided by the density $n$ at time $\Omega_{i} t=18.9$

while for the $n_{\infty}=0.2$ case it was about 0.165 .

The ion current density $j_{i, z}$ very much follows the particle density which is roughly equal for both electrons and ions. One can observe a beginning formation of secondary islands next to the diffusion region. We attribute this to the high growth rate of the instability for the low density values. At later times the secondary island coalesces with the main island.

In Figure 3 we plot the off diagonal components $P_{x z}$ and $P_{y z}$ of the electron pressure tensor divided by the density. These two components are the main origin of the inductive electric field $E_{z}$ at the X-line. Due to symmetry conditions the electric field at the X-line is given by $E_{z}=\left(m / n e^{2}\right) \partial j_{z} / \partial t-(1 / n e)\left(\partial P_{x z} / \partial x+\partial P_{y z} / \partial y\right)$. In [10] it was observed, for the case with $n_{\infty}=0.2$, that the electron inertia played only a secondary role while the two terms from the pressure tensor contributed to roughly equal amounts to the electric field. Here we see that, for the $n_{\infty}=0$ case, the contribution of the $P_{x z}$ term dominates over the $P_{y z}$ term. The maxima of $P_{x z} / n e$ are more than a factor of 6 larger than the maxima of $P_{y z} / n e$. The bar like structure of $P_{y z}$ is still seen, as in [10], but the bars are further apart, again reducing the gradient $\partial P_{y z} / \partial y$. The $P_{x z}$ originates from the bunched gyro motion of the accelerated electrons in the outflow magnetic field. On the other hand in [10] the $P_{y z}$ term originated from the electrons that were accelerated in the inflow region and crossed the neutral line due to their inertia while being accelerated in the $z$-direction. Without background density these electrons are missing and the $P_{y z}$ stays small. As a consequence the source inductive electric field is made up almost completely by the $\partial P_{x z} / \partial x$ contribution. 


\section{Summary and Conclusions}

We have performed a $2 \frac{1}{2}$-dimensional Vlasov simulation of collisionless reconnection without a background density. To allow comparison, all other parameters were chosen to be equal to the GEM setup [1]. Some differences were found that could be attributed to the lack of a background population. The onset of the fast reconnection was not influenced by the background density but the reconnection rate was found to be considerably faster when no background population was present. This increase in the reconnection rate can be attributed to the faster Alfvèn velocity as the density decreases. The faster reconnection rate causes secondary islands to form. However, no full development of secondary $\mathrm{X}$ lines could be observed as the secondary islands quickly coalesce with the main island. In previous investigations it became apparent, that on the $\mathrm{X}$-line only the off diagonal components of the electron pressure tensor carry a major contribution to the reconnection electric field. When a background density is present both the $P_{x z}$ and $P_{y z}$ contribute to almost equal amounts. Due to the lack of inflowing electrons in the $n_{\infty}=0$ case, the $P_{y z}$ is greatly reduced and only the $P_{x z}$ is dominant at the $\mathrm{X}$-line.

Acknowledgements

This work was supported by the SFB 591 of the Deutsche Forschungsgesellschaft. Access to the JUMP multiprocessor computer at Forschungszentrum Jülich was made available through project HBO20. Part of the computations were performed on an LinuxOpteron cluster supported by HBFG-108-291.

\section{References}

[1] J. Birn, J. F. Drake, M. A. Shay, B. N. Rogers, R. E. Denton, M. Hesse, M. Kuznetsova, Z. W. Ma, A. Bhattacharjee, A. Otto, P. L. Pritchett, GEM Magnetic Reconnection Challenge, J. Geophys. Res. 106 (2001) 3715.

[2] M. A. Shay, J. F. Drake, B. N. Rogers, R. E. Denton, Alfvénic collisionless magnetic reconnection and the Hall term, J. Geophys. Res. 106 (2001) 3759.

[3] R. F. Lottermoser, M. Scholer, Undriven magnetic reconnection in magnetohydrodynamics and Hall magnetohydrodynamics, J. Geophys. Res. 102 (1997) 4875.

[4] V. M. Vasyliunas, Theoretical models of magnetic field line merging, Rev. Geophys. 13 (1975) 303.

[5] J. W. Dungey, Noise-free neutral sheets, in: T. D. Guyenne, J. J. Hunt (Eds.), Reconnection in Space Plasmas, Vol. 15, Eur. Space Agency Spec. ESA, 1988, pp. SP-285.

[6] M. Hesse, D. Winske, Hybrid simulations of collisionless ion tearing, Geophys. Res. Lett. 20 (1993) 1207.

[7] M. M. Kuznetsova, M. Hesse, D. Winske, Kinetic quasi-viscous and bulk flow inertia effects in collisionless magnetotail reconnection, J. Geophys. Res. 103 (1998) 199.

[8] C. K. Birdsall, A. B. Langdon, Plasma Physics via Computer Simulation, McGraw-Hill, New York, 1985.

[9] H. Schmitz, R. Grauer, Darwin-vlasov simulations of magnetised plasmas, J. Comp. Phys. 214 (2006) 738.

[10] H. Schmitz, R. Grauer, Kinetic vlasov simulations of collisionless magnetic reconnection, Phys. Plasmas 13 (2006) 092309. 
[11] F. Filbet, E. Sonnendrücker, P. Bertrand, Conservative Numerical Schemes for the Vlasov Equation, J. Comp. Phys. 172 (2001) 166.

[12] E. G. Harris, On a Plasma Sheath Separating Regions of Oppositely Directed Magnetic Field, Il Nuovo Cimento 23 (1962) 115. 